\title{
Histopathological Spectrum of Lesions in Fallopian Tube
}

\author{
Dr Prachi Kukreja ${ }^{1}$,Dr Jayaprakash Shetty $\mathrm{K}^{2}$ \\ ${ }^{I}$ (Senior Resident, Department Of Pathology, Babu Jagjivan Ram Memorial Hospital, India) \\ ${ }_{2}^{2}$ (Professor And Head, Department Of Pathology, K.S. Hegde Medical Academy, India)
}

\begin{abstract}
Background: Fallopian tube is a common specimen in the laboratory. It is affected by a wide spectrum of diseases but there are only occasional studies, documenting histological changes in the fallopian tube removed for all reasons.

Aims and objectives:To study the gross and microscopic features of the excised fallopian tubes and to study the common and unusual lesions in them. To find the incidences of tumour and the tumour like lesions of the fallopian tube.

Materials and Methods: A sample size of 200 cases was included in this study which consisted of all the specimens of fallopian tubes of all age groups obtained by salpingectomy, tubal ligation, salpingooophorectomy or along with hysterectomy. All the specimens were processed by routine histopathological techniques. Histopathological features were studied on hematoxylin and eosin stained sections.

Results: In our study, majority of the cases belonged to age group of less than or equal to 30 years. Total number of 372 fallopian tubes were received from 200 patients. Out of the 372 fallopian tube specimens, 248 (66.67\%) were normal whereas 124 (33.33\%) fallopian tubes were abnormal. Of the 124 abnormal fallopian tube specimens, majority were diagnosed to have non neoplastic lesions that is 119 (95.97\%) fallopian tubes, and $5(4.03 \%)$ were diagnosed to have neoplastic lesions. The most common histopathological finding observed were inflammatory conditions seen in $47(12.63 \%)$ of the fallopian tubes seen most commonly in the age group of less than or equal to 30 years.

Conclusion: The present study describes the broad spectrum of lesions in fallopian tube and their incidence with respect to age group. Majority of the lesions were non neoplastic comprising predominantly of inflammatory lesions, seen most commonly in younger age group. Neoplastic lesions of the fallopian tube were rare.
\end{abstract}

Keywords: Histopathology, Fallopian tube, Salpingitis, Hyatidiform mole, Ectopic pregnancy

\section{Introduction}

The fallopian tube is a common specimen in the laboratory; it may be examined alone as salpingectomy or tubal ligation specimen or as a part of complex specimen from hysterectomy and/or oophorectomy operations. ${ }^{1}$

Although, fallopian tube are affected by a wide spectrum of diseases varying from salpingitis to carcinoma, literature search revealed that there are only occasional studies, documenting histological changes in the fallopian tube removed for all reasons. As this is a common specimen in the general surgical pathology laboratory, knowledge of the general frequency of these variations in a general pathology practice can be valuable to the pathologist who identifies a potentially unusual histological finding. It is observed that tubal pathology with inflammatory lesion forms the major group followed by tubal ectopic pregnancy which is an important cause of maternal morbidity and mortality. ${ }^{2,3}$ Primary carcinoma is a rare finding. But recent contributions have demonstrated tubal epithelium as the potential origin of tubal, ovarian and peritoneal carcinoma.

This study aims at diagnosis and finding the incidence of broad spectrum of fallopian tube lesions and to find the incidence of these diseases with respect to different age groups and spectrum of lesions associated with benign and malignant fallopian tube diseases.

\section{Materials And Methods}

The present histopathological study includes analysis of surgically removed fallopian tubes during salpingectomy, tubal ligation, or along with hysterectomy/oophorectomy. It is a prospective type of study done on a sample size of 200 cases.

The clinical notes of all cases were reviewed for the following data: patient's age, clinical history. The resected samples received were subjected to gross examination noting their size and shape including their external surface and cut surface. Representative sections were taken from each tube. Sections from formalin fixed, paraffin embedded tissues were stained with hematoxylin and eosin. 
The histopathology slides were examined and final diagnosis was made. Findings of this study were tabulated and statistically analysed.

\section{Results}

The present study was based on a sample size of 200 cases. Cases from all the age groups were analysed in the study. Various lesions in fallopian tube were diagnosed by detailed histopathological examination of tissue sections. The salient observations noted in the study were:

\section{In the present study, majority of the patients were less than or equal to 30 years. (Table 1)}

The most common clinical symptom with which the patients presented was pain abdomen comprising of 98 cases (49\%). Out of these 98 cases, 41 cases $(20.5 \%)$ were pregnant females who presented with labour pains, 34 cases $(17 \%)$ cases had clinically suspected ovarian or uterine pathology and the remaining 23 cases $(11.5 \%)$ had other clinical diagnosis like ectopic pregnancy, hydrosalpinx, paratubal cyst, adnexal mass and tubo-ovarian mass. The other common clinical symptom was menorrhagia comprising of 35 cases (17.5\%), 12 cases $(6 \%)$ presented with spotting per vaginam and 8 cases $(4 \%)$ presented with post-menopausal bleeding. In most of these cases the clinical symptoms were due to pathological condition affecting the ovary or the uterus and fallopian tubes were removed along with them in surgical procedures like hysterectomy and/or oophorectomy.

In majority of the patients, the clinical indication was tubal sterilization comprising of 103 cases $(51.5 \%)$. Out of these 103 cases, 61 cases $(30.5 \%)$ were the pregnant females who underwent tubal sterilization concurrent with caesarean section, the other 41 cases $(20.5 \%)$ underwent sterilization following a vaginal delivery and the remaining 1 case $(0.5 \%)$ was a pregnant female who underwent dilatation and curettage with tubal sterilization. (Table 2)

Of the 200 cases, unilateral fallopian tubes were removed in 28 cases, whereas in other 172 cases bilateral fallopian tubes were removed. Therefore, total number of 372 fallopian tubes were received from 200 patients. Out of the 372 fallopian tube specimens, 248 (66.67\%) were normal whereas 124 (33.33\%) fallopian tubes were abnormal, that is these tubes had a non-neoplastic or a neoplastic lesion. (Table 3) Of the 124 abnormal fallopian tube specimens, majority were diagnosed to have non neoplastic lesions is 119 (95.97\%), and $5(4.03 \%)$ were diagnosed to have neoplastic lesions. Out of the 372 fallopian tube specimens, majority were grossly unremarkable that is $288(77.42 \%)$ specimens. The most common gross finding was dilated lumen seen in $33(8.87 \%)$ fallopian tubes.

In the 372 fallopian tube specimens, the most common histopathological finding observed were inflammatory conditions seen in $47(12.63 \%)$ of the fallopian tubes. The inflammatory lesions comprised of salpingitis in $40(10.75 \%)$, pyosalpinx in $2(0.54 \%)$ and hydrosalpinx in $7(1.88 \%)$ of fallopian tube specimens. Out of 40 tubes showing salpingitis, acute salpingitis was most common, present in $21(5.64 \%)$ of the specimens. This was followed by paratubal cyst $14(3.76 \%)$ and walthard cell nests 12 (3.22\%) of fallopian tubes. Other lesions observed were haematosalpinx seen in 7 (1.88\%) fallopian tube specimens, tubal ectopic pregnancy in 5 tubes $(1.34 \%)$, tubal epithelial hyperplasia in 5 tubes (1.34\%), pyosalpinx, endometriosis, salpingitis isthmica nodosa, hydatidiform mole and secondary malignancy were seen in $2(0.54 \%)$ of specimens each. Decidual reaction, exaggerated placental site reaction and primary carcinoma of fallopian tube were seen 1 fallopian tube $(0.27 \%)$ each. (Table 4, Figure 1, Figure 2, Figure 3) Also seen were $21(5.65 \%)$ of the fallopian tubes having several combinations of non-neoplastic lesions.

Tubercular salpingitis was an incidental finding in a Medico legal autopsy where, the deceased had alleged history of road traffic accident.In the fallopian tube specimens with histopathological findings, salpingitis was the most common lesion seen commonly in the age group of less than or equal to 30 years. Acute salpingitis was the most common type of salpingitis, also seen commonly in the same age group. Out of the 13 tubal ectopic pregnancy, majority of the cases belonged to the age group of 21-30 years comprising of 8 cases $(61.54 \%)$. There were 5 cases $(38.46 \%)$ belonging to the age group 31-40 years. No case was seen after 40 years of age. There were two cases of tubal ectopic pregnancy which histologically showed hydatidiform mole, belonging to the age groups of less than or equal to 30 and 31-40 years. The 13 patients who were diagnosed to have tubal ectopic pregnancy presented clinically with either pain abdomen or spotting per vaginam or both. Pain abdomen was present in 10 cases $(76.92 \%)$ and spotting per vaginam in 8 cases $(61.54 \%)$. The two cases of tubal ectopic pregnancy diagnosed as hydatidiform mole presented with both pain abdomen and spotting per vaginam.

Of the 13 patients with tubal ectopic pregnancy majority of the patients had history of previous child birth comprising of 10 cases $(76.92 \%)$ out of which 5 cases $(38.46 \%)$ had parity of more than or equal to 2 and other 5 cases $(38.46 \%)$ had parity of 1 . There were 3 cases $(23.08 \%)$ which were nullipara. History of abortion was seen in 3 cases $(23.07 \%)$ of which 1 case $(7.69 \%)$ had history of two or more abortions whereas the other 2 cases $(15.38 \%)$ had history of one abortion. Other 10 cases $(76.93 \%)$ had no abortion history. Out of the two 
cases of tubal ectopic pregnancy diagnosed as hydatidiform mole one of the case had history of previous one abortion. Tubal ectopic pregnancy was most commonly seen in right fallopian tube comprising of 9 cases $(69.23 \%)$. Remaining 4 cases $(30.77 \%)$ were seen in the left fallopian tube.

The 13 cases of tubal ectopic pregnancy (including 2 cases of hydatidiform mole), some other histopathological findings were present in the same specimen in 6 cases $(46.15 \%)$. Chronic salpingitis was present in 2 cases $(15.39 \%)$ followed by 1 case $(7.69 \%)$ each of tubal ectopic pregnancy with walthard cell nests or paratubal cyst or salpingitis isthmica nodosa or endometriosis.

\section{Discussion}

In the present study, the clinical history, gross and histopathological examination of all the 372 fallopian tube specimens from 200 cases were collected and analysed. Out of 372 fallopian tubes 248 were normal and 124 abnormal, that is these tubes had a non-neoplastic or a neoplastic lesion. Out of the 124 abnormal fallopian tube specimens, 119 had non neoplastic lesions and the remaining 5 had neoplastic lesions. The most common histopathological finding observed in non-neoplastic lesions were inflammatory conditions seen in 49 fallopian tubes. The inflammatory lesions comprised of salpingitis in 40, pyosalpinx in 2 and hydrosalpinx in 7 of fallopian tube specimens. Out of 40 tubes showing salpingitis, acute salpingitis was most common present in 21 tubes, seen commonly in the age group of less than or equal to 30 years.

In the study conducted by IN Bagwan et al $(2004)^{[2]}$, majority of the cases belonged to the age group of $36-45$ years. Gon $\mathrm{S}$ et al (2013) ${ }^{[1]}$ showed same findings with majority of the cases belonging to the age group of 36-45 years. In the present study, most of the patients were less than or equal to 30 years.

In study conducted by Jennifer L. Hunt et al (2002) ${ }^{[4]}$, tubal ligation and total abdominal hysterectomy with bilateral salpingo-oophorectomy were done in $38.62 \%$ of the cases each followed by salpingectomy and unilateral salpingo-oophorectomy done in $22.76 \%$ of the cases. In IN Bagwan et al (2004) ${ }^{[2]}$, most common surgical procedure performed was total abdominal hysterectomy with bilateral salpingo-oophorectomy in $72.77 \%$ of the cases followed by salpingo-oophorectomy in $22.9 \%$ cases and tubal ligation in $4.24 \%$ cases. In Jha $\mathrm{R}$ et al (2006) ${ }^{[5]}$, total abdominal hysterectomy with bilateral salpingo-oophorectomy was done in $56.12 \%$ cases and total abdominal hysterectomy with unilateral salpingo-oophorectomy in $5.88 \%$ cases. In Gon S et al $(2013)^{[1]}$, total abdominal hysterectomy with bilateral salpingo-oophorectomy was done in $75.11 \%$ cases followed by salpingectomy in $14 \%$ cases, unilateral salpingo-oophorectomy in $5.86 \%$ cases and tubal ligation in $5 \%$ cases. In the present study, tubal ligation was done in $51.5 \%$ cases followed by total abdominal hysterectomy with bilateral salpingo-oophorectomy in 33\% cases, salpingectomy in $9 \%$ cases, unilateral salpingo-oophorectomy in 3\% cases, total abdominal hysterectomy with unilateral salpingo-oophorectomy in $2 \%$ cases and bilateral salpingo-oophorectomy in $1.5 \%$ cases.

In the present study, the percentage of normal fallopian tube specimens was $66.67 \%$. Study done by IN Bagwan et al $(2004)^{[2]}$ and Gon S et al $(2013)^{[1]}$ showed similar findings with percentage of normal fallopian tube specimens being $66.52 \%$ and $69 \%$ respectively. Study conducted by Jha R et al $(2006)^{[5]}$ showed a higher percentage of normal fallopian tubes that is $84.3 \%$ of the specimens. Our study was in concordance with other studies.

In study conducted by Jennifer L. Hunt et al $(2002)^{[4]}$, most common lesions were non neoplastic in which inflammatory lesions such as salpingitis was most common, seen in $32.5 \%$ of the specimens followed by tubal epithelial hyperplasia seen in $10.8 \%$ of the specimens. In IN Bagwan et al (2004) ${ }^{[2]}$, also the most common lesions were non neoplastic lesions, in which inflammatory lesions were the most common seen in $18.34 \%$ tubes. The inflammatory lesions here comprised of salpingitis in $10.19 \%$, pyosalpinx in $0.29 \%$ and hydrosalpinx in $7.86 \%$ of fallopian tube specimens. In Jha R et al $(2006)^{[5]}$, non-neoplastic lesions were most common lesions in which paratubal cyst was most common seen in $6.1 \%$ of the specimens closely followed by inflammatory lesions that is salpingitis in $5.4 \%$ of the specimens. In study conducted by Gon $\mathrm{S}$ et al $(2013)^{[1]}$, ectopic pregnancy was the most common finding seen in $13.5 \%$ of the specimens closely followed by inflammatory lesions in $13.35 \%$ of the specimens comprising of salpingitis in $12.66 \%$ and hydrosalpinx in $0.69 \%$. In the present study, the most common lesions were also non neoplastic lesions, in which inflammatory lesions were most commonly seen in $13.17 \%$ of the fallopian tube specimens. The inflammatory lesions comprised of salpingitis in $10.75 \%$, pyosalpinx in $0.54 \%$ and hydrosalpinx in $1.88 \%$ of fallopian tube specimens. This was followed by paratubal cyst in $3.76 \%$ of the specimens. The present study showed concordance with other studies. However, in the study conducted by Gon S et al (2013) ${ }^{[1]}$, ectopic pregnancy was most common lesion closely followed by inflammatory lesions, this was because more cases were included in this study and all the specimens of ectopic pregnancy were sent for histopathological examination from the gynaecology department as compared to other specimens.

In all the studies neoplastic lesions of fallopian tube are rare finding. In study conducted by Jennifer L. Hunt et al $(2002)^{[4]}$, metastatic lesions were seen in $1.4 \%$ of the specimens. In IN Bagwan et al $(2004)^{[2]}$, only malignant lesions were seen out of which primary malignancy comprised of $0.15 \%$ of tubes and secondary 
malignancy was seen in $0.29 \%$ of tubes. Jha $\mathrm{R}$ et al $(2006)^{[5]}$ reported secondary malignancies in $3.4 \%$ of the specimens. In study conducted by Gon S et al $(2013)^{[1]}$, benign lesions were seen in $0.03 \%$ of specimens, primary malignancy in $0.03 \%$ and secondary in $0.20 \%$ of the specimens. In the present study benign lesions were seen in $0.54 \%$ specimens, primary malignancy in $0.27 \%$ of specimens and secondary malignancy in $0.54 \%$ of specimens.

In the present study, most cases with fallopian tubes having acute salpingitis were of the age 30 years or younger which was in concordance with study done by IN Bagwan et al (2004) ${ }^{[2]}$ which also showed acute salpingitis in younger age group that is $15-30$ years. In study done by N. Dahiya et al $(2011)^{[6]}$, tubal ectopic pregnancy was commonly seen in younger age group of 25-29 years. In Shraddha Shetty K et al (2014) ${ }^{[3]}$, it was seen in 25-30 years and in C. Li et al (2014) ${ }^{[7]}$, most common age group was 20-29 years. In the present study, tubal ectopic pregnancy was also seen in younger age group of $21-30$ years.

In study conducted by Linda K. Green et al $(1989)^{[8]}$, majority of tubal ectopic pregnancy had varied histopathological finding associated with it in which the most common was chronic salpingitis seen in $88 \%$ of the cases followed by salpingitis isthmica nodosa in $43 \%$ of cases. In N. Dahiya et al $(2011)^{[6]}$, the most common associated histopathological finding was chronic salpingitis seen in $39 \%$ of the cases followed by acute salpingitis in $18 \%$ and salpingitis isthmica nodosa in $6 \%$ of the cases. The present study was in concordance with above two studies with chronic salpingitis being the most common associated histopathological finding with tubal ectopic pregnancy, seen in $15.39 \%$ of the cases followed by salpingitis isthmica nodosa, endometriosis, walthard cell nests and paratubal cyst, seen in $7.69 \%$ of the cases each.

\section{Conclusion}

Fallopian tube is a common specimen in the surgical pathological laboratory. It is resected alone as salpingectomy or tubal ligation specimen or along with other surgical pathological specimens like hysterectomy and/or oophorectomy.

The present study describes the broad spectrum of lesions in fallopian tube and their incidence with respect to age group. Majority of the lesions were non neoplastic comprising predominantly of inflammatory lesions, seen most commonly in younger age group. Neoplastic lesions of the fallopian tube were rare.

Also seen were some rare presentations like a case of tubercular salpingitis which was an incidental finding in a Medico legal autopsy, where the deceased had alleged history of road traffic accident. As fallopian tube is a common specimen in laboratory therefore knowledge of various lesions is valuable for the pathologists and histopathological examination of fallopian tube specimens is necessary for documentation and diagnosis as many lesions can be missed clinically which might have direct bearing on the patient's further management and follow up.

\section{References}

[1]. Gon S, Basu A, Majumdar B, Das TK, Sengupta M, Ghosh D. Spectrum of histopathological lesions in the fallopian tubes. J Pathol Nepal. 2013;3:356 -60.

[2]. Bagwan IN, Harke AB, Malpani MR, Deshmukh SD. Histopathological spectrum of lesions encountered in the fallopian tube. J Obstet Gynecol Ind. 2004 Jul-Aug;54(4):379-82

[3]. K SS, K AS. Clinical study of ectopic pregnancies in a tertiary care hospital of Mangalore. Innov J Med Health Sci. 2014 JanFeb;4:305-9.

[4]. Hunt JL, Lynn AA. Histologic features of surgically removed fallopian tubes. Arch Pathol Lab Med. 2002 Aug;126(8):951-5.

[5]. Jha R, Pant AD, Jha A, Adhikari RC, Sayami G. Histopathological analysis of hysterectomy specimens. JNMA J Nepal Med Assoc. 2006 Jul-Sep;45(163):283-90.

[6]. N. Dahiya, S. Singh, R. Kalra, R. Sen and S. Kumar. Histopathological changes associated with ectopic tubal pregnancy. IJPSR 2011;2(4):929-33.

[7]. Li C, Meng CX, Zhao WH, Lu HQ, Shi W, Zhang J. Risk factors for ectopic pregnancy in women with planned pregnancy: a casecontrol study. Eur J Obstet Gynecol Reprod Biol. 2014 Oct;181:176-82.

[8]. Green LK, Kott ML. Histopathological findings in ectopic tubal pregnancy. Int J Gynecol Pathol.1989;8:255-62

Tables and Figures

\begin{tabular}{|c|c|c|c|}
\hline \multicolumn{4}{|c|}{ Table 1- Showing age distribution of the patients } \\
\hline SL NO. & Age (years) & Frequency & Percentage (\%) \\
\hline 1$)$ & $<=30$ & 85 & 42.5 \\
\hline 2$)$ & $31-40$ & 54 & 27 \\
\hline 3$)$ & $41-50$ & 43 & 21.5 \\
\hline 4$)$ & $51-60$ & 10 & 5.0 \\
\hline 5$)$ & Above 60 & 08 & 4.0 \\
\hline & Total & 200 & 100 \\
\hline
\end{tabular}

\begin{tabular}{|c|l|c|c|}
\hline \multicolumn{2}{|c|}{ Table 2- Showing distribution of cases according to the type of surgery performed } \\
\hline SL NO. & \multicolumn{1}{|c|}{ Surgical Procedure } & Frequency & $\%$ \\
\hline 1$)$ & Salpingectomy & 18 & 9.0 \\
\hline 2$)$ & Unilateral salpingo-oophorectomy & 06 & 3.0 \\
\hline
\end{tabular}




\begin{tabular}{|c|l|c|c|}
\hline 3$)$ & Bilateral salpingo-oophorectomy & 03 & 1.5 \\
\hline 4$)$ & $\begin{array}{l}\text { Total abdominal hysterectomy with } \\
\text { unilateral salpingo-oophorectomy }\end{array}$ & 04 & 2.0 \\
\hline 5$)$ & $\begin{array}{l}\text { Total abdominal hysterectomy with } \\
\text { bilateral salpingo-oophorectomy }\end{array}$ & 66 & 33.0 \\
\hline 6$)$ & Tubal ligation & 200 & 51.5 \\
\hline & Total & & 100.0 \\
\hline
\end{tabular}

\begin{tabular}{|c|l|c|c|}
\hline \multicolumn{4}{|c|}{ Table 3- Showing incidence of non-neoplastic and neoplastic lesions in the total number } \\
of abnormal fallopian tube specimens
\end{tabular}

Table 4- Showing distribution of various tubal lesions

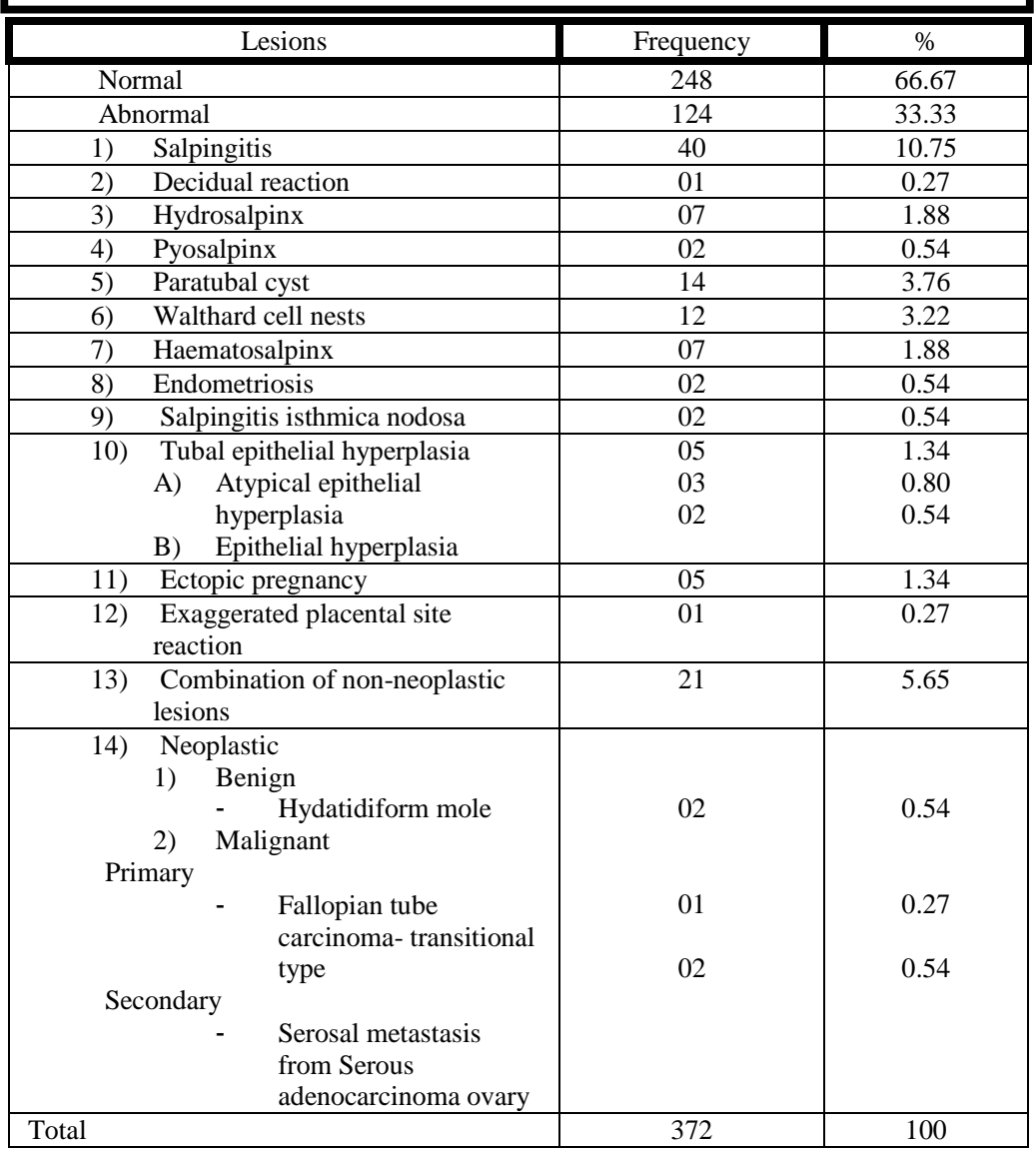

Figure 1- Pie chart showing incidence of various tubal lesions

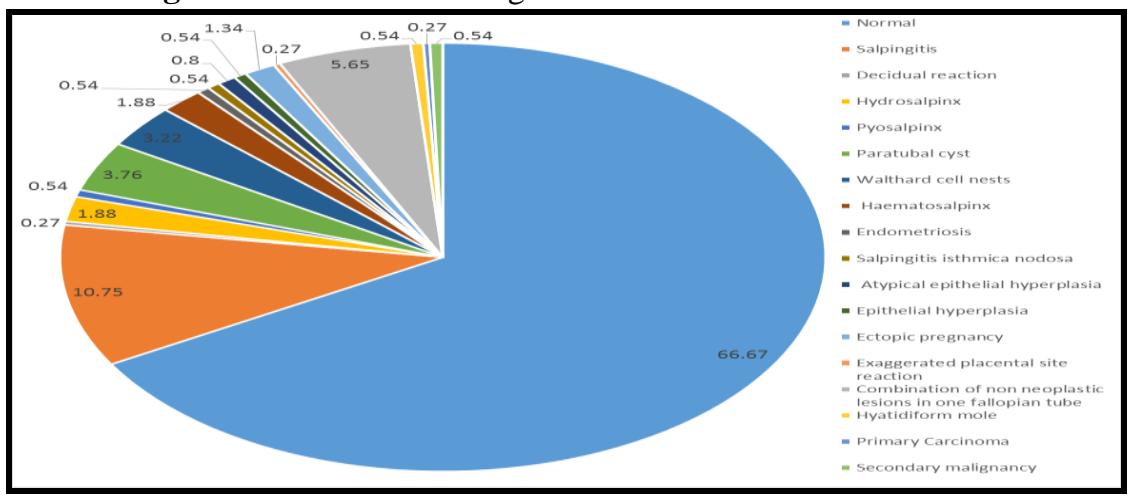




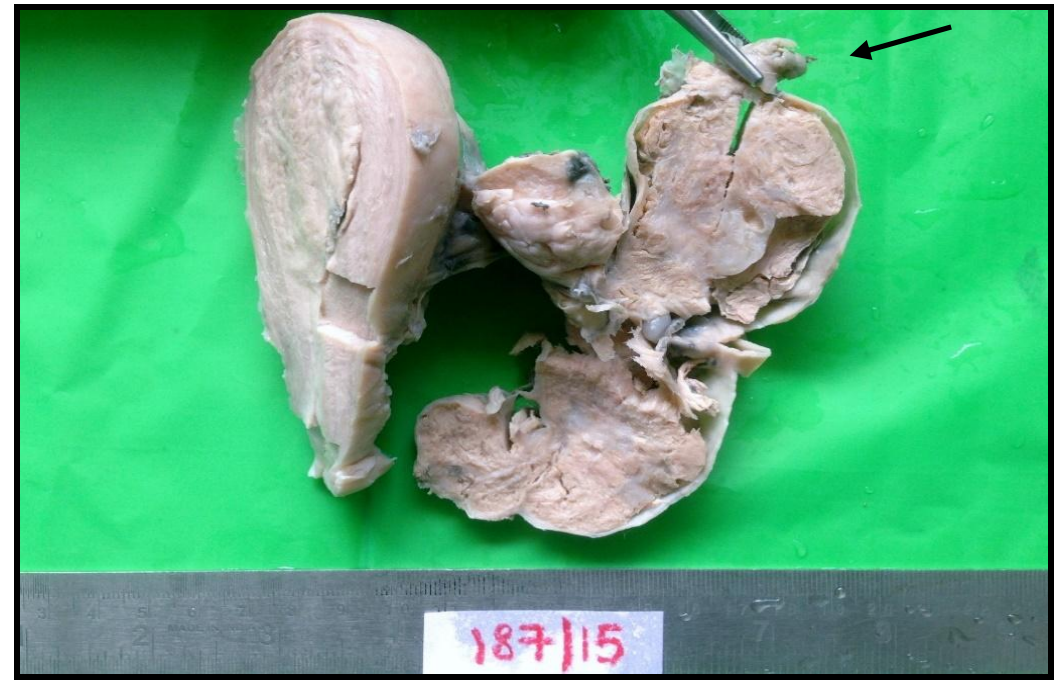

Figure 2 - Gross photograph showing tubal lumen distended with grey white mass. Tubal fimbriae seen at periphery (arrow).

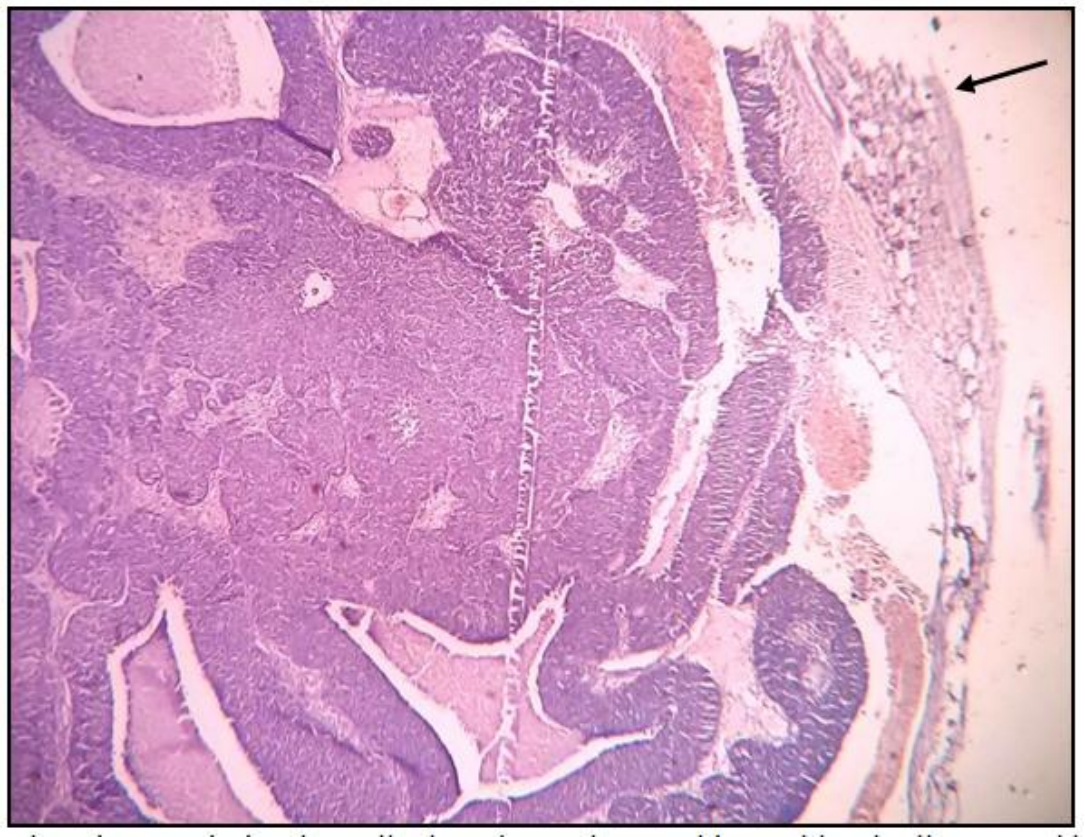

Figure 3 - Microphotograph showing Fallopian tube carcinoma with transitional cells arranged in papillary structures, glands and nests showing stratification and loss of polarity. Tubal wall seen in periphery (arrow). (H\&E 4X) 